\title{
Conjugate free convection over a vertical slender hollow cylinder embedded in a porous medium
}

\author{
I. Pop, T.-Y. Na
}

\begin{abstract}
A numerical study of the steady conjugate free convection over a vertical slender, hollow circular cylinder with the inner surface at a constant temperature and embedded in a porous medium is reported. The governing boundary layer equations for the fluid-saturated porous medium over the cylinder along with the one-dimensional heat conduction equation for the cylinder are cast into dimensionless form, by using a non-similarity transformation. The resulting non-similarity equations with their corresponding boundary conditions are solved by using the Keller box method. Emphasis is placed on the effects caused by the wall conduction parameter, $p$, and calculations have covered a wide range of this parameter. Heat transfer results including the temperature profiles, the interface temperature profiles and the local Nusselt number are presented.
\end{abstract}

\section{List of symbols}

$F$ reduced stream function for $p=0$ (non-conjugate problem)

$f \quad$ reduced stream function for $p \neq 0$

$g$ acceleration due to gravity

$\mathrm{h}_{x} \quad$ local heat transfer coefficient

$k$ thermal conductivity

$k_{m}$ effective thermal conductivity of the saturated porous medium

$K$ permeability of saturated porous medium

$L \quad$ length of the cylinder

$\mathrm{Nu}_{x}$ local Nusselt number

$p \quad$ conjugate conduction parameter defined in Eq. (18)

$\dot{q}$ heat transfer

$r$ radial coordinate

$r_{i}, r_{o}$ inner and outer radii of the hollow cylinder

$\mathrm{Ra}$ modified Rayleigh number

$\mathrm{Ra}_{x}$ modified local Rayleigh number

$T$ temperature

$T_{b}$ temperature at the inner surface of the hollow cylinder

$T_{s}$ temperature of the hollow cylinder

Received on 17 November 1997

Ioan Pop

Faculty of Mathematics

University of Cluj, R-3400 Cluj, CP 253, Romania

Tsung-Yen $\mathrm{Na}(\square)$

Dept. of Mechanical Engineering

University of Michigan-Dearborn

Dearborn, MI, 48128, USA

$\Delta T$ reference temperature

$u_{c}$ reference velocity

$v_{r} \quad$ velocity component in r-direction

$v_{x} \quad$ velocity component in $\mathrm{x}$-direction

$x \quad$ coordinate along the axis

$y$ independent variable defined as $y=r-r_{\mathrm{o}}$

\section{Greek symbols}

$\alpha \quad$ effective thermal diffusivity of the saturated porous medium

$\beta \quad$ thermal expansion coefficient

$\xi \quad$ transformed variable defined in Eq. (23)

$\eta \quad$ non-similarity variable

$\theta$ dimensionless temperature

$v \quad$ kinematic viscosity

$\psi \quad$ stream function

\section{Subscripts \\ $w$ wall condition \\ $s$ solid \\ $\infty$ ambient condition}

\section{Superscripts \\ dimensionless variables \\ transformed variables \\ differentiation with respect to $\eta$}

1

\section{Introduction}

The heat transfer mode in porous media has great practical importance in geophysics and energy related engineering problems. These include the utilization of geothermal energy, the control of pollutant spread in ground-water, the design of nuclear reactors, compact heat exchangers, solar power collectors, high performance insulation for buildings, food processing, casting and welding of a manufacturing process, etc. A detailed review of the subject of convective flow in porous media, including an exhaustive list of references, was recently performed by Nield and Bejan[1], and Ingham and Pop [2].

In many practical problems the information on the interfacial temperature is essential because the heat transfer characteristics are mainly determined by the temperature differences between the bulk flow and the interface. These are usually referred to as conjugate heat transfer problems, and they have many practical applications, particularly those related to energy conservation in buildings. Conju- 
gate heat transfer from vertical or horizontal surfaces as well as horizontal cylinders embedded in porous media have recently been extensively investigated [3-11], and a detailed literature survey was most recently provided by Kimura et al. [12]. However, the problem of conjugate heat transfer over a vertical cylinder in a porous medium has not yet received any attention. Therefore, the present study proposes a mathematical model to investigate the conjugate problem of free convection over the outside surface of a vertical slender, hollow circular cylinder which is embedded in a porous medium. The temperature of the inner surface of the cylinder is kept at a constant value $T_{b}$ and the temperature of the outer surface is determined by the conjugate solution of the energy equation of the solid and the boundary layer equations of the fluid-saturated porous medium. The approximation of the one-dimensional conduction term (transversal) in the energy equation of the cylinder is taken into account because the aspect ratio $r_{o} / L$ (outer radius/length) is sufficiently small in this study. Due to close coupling for this problem, the boundary layer equations outside the cylinder and the onedimensional heat conduction equation for the hollow cylinder must simultaneously be solved. These equations being non-similar are solved by a very efficient finite-difference method known as Keller-box scheme [13]. This solution methodology has been well established in a recent paper by $\mathrm{Na}$ [14] for the corresponding problem of a viscous (non-porous) fluid. However, it is worth mentioning that neither the surface temperature nor the heat flux through the wall of the hollow cylinder is known a priori. Thus, an iterative procedure would have to be applied. The effects of the conduction parameter, $p$, on the heat transfer characteristics are discussed.

\section{2}

\section{Basic equations}

The geometry considered is a vertical slender, hollow circular cylinder with inner and outer radii $r_{i}$ and $r_{o}$,

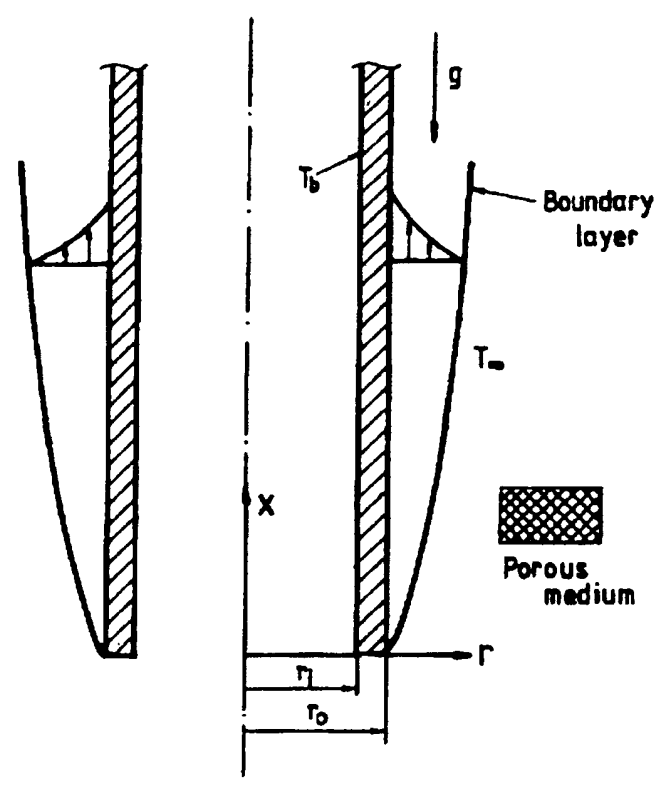

Fig. 1. Physical model and coordinate system respectively, which embedded in a fluid-saturated porous medium as shown in Fig. 1. The inner surface of the cylinder is held at a constant temperature $T_{b}$, while the temperature of the ambient fluid is $T_{\infty}$, where $T_{b}>T_{\infty}$. The porous material is isotropic and homogeneous, and the fluid is incompressible. Under these assumptions, the governing boundary layer equations for the conjugate free convection over a vertical slender, hollow circular cylinder embedded in a porous medium based on Darcy-Bous-

sinesq approximation are:

for the fluid-porous medium

$\frac{\partial\left(r v_{x}\right)}{\partial x}+\frac{\partial\left(r v_{r}\right)}{\partial r}=0$

$v_{x}=\frac{g \beta K}{v}\left(T-T_{\infty}\right)$

$v_{x} \frac{\partial T}{\partial x}+v_{r} \frac{\partial T}{\partial r}=\frac{\alpha}{r} \frac{\partial}{\partial r}\left(r \frac{\partial T}{\partial r}\right)$

for the solid cylinder

$\frac{1}{r} \frac{\partial}{\partial r}\left(r \frac{\partial T_{s}}{\partial r}\right)+\frac{\partial^{2} T_{s}}{\partial x^{2}}=0$

If Eq. (4) is normalized by introducing the dimensionless variables

$\bar{r}=\frac{r}{r_{o}}, \quad \bar{x}=\frac{x}{L}, \quad \theta_{s}=\frac{\left(T_{s}-T_{\infty}\right)}{\Delta T}$

Where $\Delta T=T_{b}-T_{\infty}$, we then get

$\frac{1}{\bar{r}} \frac{\partial}{\partial \bar{r}}\left(\bar{r} \frac{\partial \theta_{s}}{\partial \bar{r}}\right)+\left(\frac{r_{\mathrm{o}}}{L}\right)^{2} \frac{\partial^{2} \theta_{s}}{\partial \bar{x}^{2}}=0$

Assuming that $r_{o} / L \ll 1$, then the axial conduction temperature, i.e., $\partial^{2} T_{s} / \partial x^{2}$, can be neglected, so that Eq. (6) becomes

$\frac{\partial}{\partial r}\left(r \frac{\partial \theta_{s}}{\partial r}\right)=0$

subject to

$r=r_{i}: T_{s}=T_{b} ; \quad r=r_{o}: T_{s}=T\left(x, r_{o}\right)$

The solution of Eq. (7) along with (8) is

$T_{s}=T_{b}+\left(T\left(x, r_{o}\right)-T_{b}\right) \frac{\ln \left(r / r_{i}\right)}{\ln \left(r_{o} / r_{i}\right)}$

On the other hand, we have that at the surface of the cylinder the heat flux from the solid and fluid-porous medium interface are equal, i.e.,

$r=r_{o}:-k_{s} \frac{\partial T_{s}\left(r_{o}\right)}{\partial r}=-k_{m} \frac{\partial T\left(x, r_{o}\right)}{\partial r}$

which, on using (9), becomes

$y=0:-k_{s} \frac{T\left(x, r_{o}\right)-T_{b}}{r_{o} \ln \left(r_{o} / r_{i}\right)}=-k_{m} \frac{T\left(x, r_{o}\right)}{\partial y}$

where $y$ is defined as $y=r-r_{o}$. The rest of the boundary conditions for Eqs. (1) to (3) are 
$y=0: v_{r}(x, 0)=0$

$y \rightarrow \infty: v_{x}(x, \infty)=0, T(x, \infty)=T_{\infty}$

Further, we introduce the following dimensionless variables

$\bar{x}=\frac{x}{r_{o}}, \bar{y}=\frac{y}{r_{o}} \sqrt{\mathrm{Ra}}, \bar{v}_{x}=\frac{v_{x}}{u_{c}}, \bar{v}_{r}=\frac{v_{r}}{u_{c}} \sqrt{\mathrm{Ra}}$

$\theta=\frac{T-T_{\infty}}{\Delta T}, u_{c}=\frac{g K \beta \Delta T}{v}$

where $\mathrm{Ra}=u_{c} r_{o} / \alpha$ is the modified Rayleigh number for a porous medium. Equations (1) to (3) become

$\frac{\partial}{\partial \bar{x}}\left(\bar{r} \bar{v}_{x}\right)+\frac{\partial}{\partial \bar{y}}\left(\bar{r} \bar{v}_{r}\right)=0$

$\bar{v}_{x}=\theta$

$\bar{v}_{x} \frac{\partial \theta}{\partial \bar{x}}+\bar{v}_{r} \frac{\partial \theta}{\partial \bar{y}}=\frac{1}{\bar{r}} \frac{\partial}{\partial \bar{y}}\left(\bar{r} \frac{\partial \theta}{\partial \bar{y}}\right)$

subject to the boundary conditions

$\bar{v}_{r}(\bar{x}, 0)=0, \theta(\bar{x}, 0)-1=p \frac{\partial \theta(\bar{x}, 0)}{\partial \bar{y}} ;$

$\bar{v}_{x}(\bar{x}, \infty)=0, \theta(\bar{x}, \infty)=0$

where $p$ is the conjugate conduction parameter given by

$p=\frac{k_{m}}{k_{s}} \mathrm{Ra}^{1 / 2} \ln \frac{r_{o}}{r_{i}}$

Let us next introduce the Mangler transformation

$\mathrm{d} x^{*}=\mathrm{d} \bar{x}, \quad \mathrm{~d} y^{*}=\bar{r} \mathrm{~d} \bar{y}$

and the stream function, $\bar{\psi}$, defined by

$\bar{r} \bar{v}_{x}=\frac{\partial \bar{\psi}}{\partial \bar{y}}, \quad \bar{r} \bar{v}_{r}=-\frac{\partial \bar{\psi}}{\partial \bar{x}}$

Equations (15) and (16), with $\theta=\partial \bar{\psi} / \partial y^{*}$, now become

$\frac{\partial \bar{\psi}}{\partial y^{*}} \frac{\partial^{2} \bar{\psi}}{\partial x^{*} \partial y^{*}}-\frac{\partial \bar{\psi}}{\partial x^{*}} \frac{\partial^{2} \bar{\psi}}{\partial y^{* 2}}=\frac{\partial}{\partial y^{*}}\left\{\left(1+\frac{2 y^{*}}{\sqrt{\mathrm{Ra}}}\right) \frac{\partial^{2} \bar{\psi}}{\partial y^{* 2}}\right\}$

subject to the boundary conditions

$\bar{\psi}\left(x^{*}, 0\right)=0, \frac{\partial \bar{\psi}\left(x^{*}, 0\right)}{\partial y^{*}}-1=p \frac{\partial^{2} \bar{\psi}\left(x^{*}, 0\right)}{\partial y^{* 2}}$,

$\frac{\partial \bar{\psi}\left(x^{*}, \infty\right)}{\partial y^{*}}=0$

To get Eq. (21) amenable for numerical integration, we introduce the following transformation

$f(\xi, \eta)=\frac{\bar{\psi}}{x^{* 2 / 3}}, \quad \eta=\frac{y^{*}}{x^{* 1 / 3}}, \quad \xi=\frac{2 x^{* 1 / 3}}{\sqrt{\mathrm{Ra}}}$

We then obtain

$\left\{(1+\xi \eta) f^{\prime \prime}\right\}^{\prime}+\frac{2}{3} f f^{\prime \prime}-\frac{1}{3} f^{\prime 2}=\frac{1}{3} \xi\left(f^{\prime} \frac{\partial f^{\prime}}{\partial \xi}-f^{\prime \prime} \frac{\partial f}{\partial \xi}\right)$ subject to the boundary conditions

$f(\xi, 0)=0, \frac{\sqrt{\mathrm{Ra}}}{2} \xi f^{\prime}(\xi, 0)-1=p f^{\prime \prime}(\xi, 0) ;$

$f^{\prime}(\xi, \infty)=0$

where primes denote differentiation with respect to $\eta$.

The dimension less temperature on the surface of the cylinder can be written as

$\theta(\bar{x}, 0)=\frac{\sqrt{\mathrm{Ra}}}{2} \xi f^{\prime}(\xi, 0)$

where

$\bar{x}=\frac{x}{r_{o}}=\left\{\frac{\xi \sqrt{\mathrm{Ra}}}{2}\right\}^{3}$

Let us consider the effect of conduction on the rate of heat transfer. First, the local rate of heat transfer is given by

$\dot{q}_{w}(x)=-k_{m} \frac{\partial T(x, 0)}{\partial y}=k_{m} \frac{\Delta T}{r_{o}} \mathrm{Ra}^{1 / 2}\left[-f^{\prime \prime}(\xi, 0)\right]$

On the other hand, the overall local heat transfer coefficient, $h_{x}$, is given by

$h_{x}=\frac{\dot{q}_{w}(x)}{\Delta T}$

and the local Nusselt number at the outer surface of the hollow cylinder is defined as

$\mathrm{Nu}_{x}=\frac{h_{x} x}{k_{m}}$

Using (28), (29) and (30), we get

$\frac{\mathrm{Nu}_{x}}{\mathrm{Ra}_{x}^{1 / 2}}= \begin{cases}-F^{\prime \prime}(\xi, 0) & \text { for } p=0 \\ \bar{x}^{1 / 2}\left[-f^{\prime \prime}(\xi, 0)\right] & \text { for } p \neq 0\end{cases}$

3

\section{Results and discussion}

We first notice from this study that for a hollow cylinder, where the temperature on the inner surface $\left(r=r_{i}\right)$ is maintained at a constant value of $T_{b}$, the temperature on the outer surface of the cylinder $\left(r=r_{o}\right)$ given by Eq. (26) depends on the coupled solution of the conduction across the cylinder and the natural convection of the fluid-saturated porous medium over the cylinder. Intuitively, if the heat conductivity of the hollow cylinder is very large, its temperature can be expected uniform at $T_{b}$ and therefore a solution of the natural convection based on a constant wall temperature will be acceptable. An inspection of the definition of the conduction parameter $p$ reveals that the same conclusion is true if the effective thermal conductivity of the saturated porous medium is very small and/or if the thickness of the hollow cylinder is very thin.

Equation (24) subject to the boundary conditions (25) has been solved numerically using the Keller box method as described by $\mathrm{Na}$ [14]. Solutions are generated for a range of values of the axial non-dimensional coordinate $\bar{x}$ and three values of the conjugate convection parameter 
$p=0.0$ (non-conjugate problem), 1.0 and 2.0. It should be noted that the solution of the basic case $p=0.0$, where the cylinder's wall temperature is assumed to be uniform at $T_{b}$, corresponds to the problem of free convection from an isothermal vertical cylinder embedded in a porous medium first considered by Minkowycz and Cheng [15].

Fig. 2-4 show the non-dimensional temperature profiles within the boundary layer plotted against $\eta$ for some values of $\bar{x}$ when $p=0.0,1.0$ and 2.0. These figures show that the temperature profiles increase with the increase of the

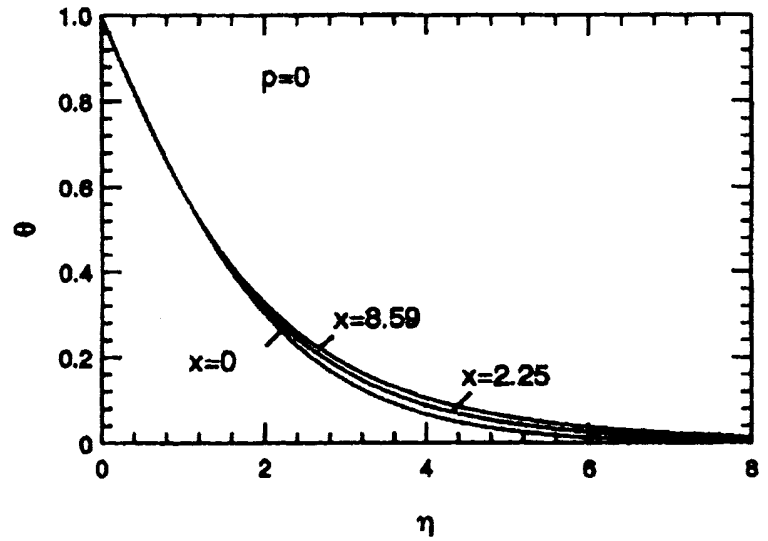

Fig. 2. Temperature profiles for $p=0.0$ (non-conjugate problem)

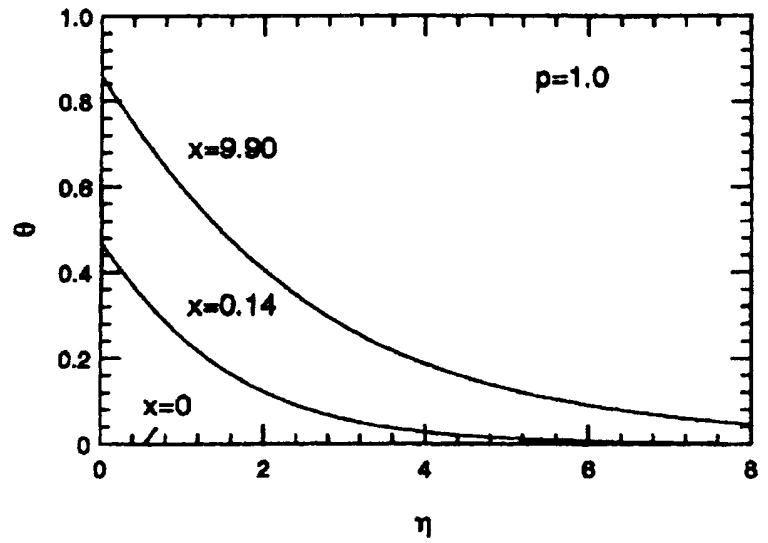

Fig. 3. Temperature profiles for $p=1.0$

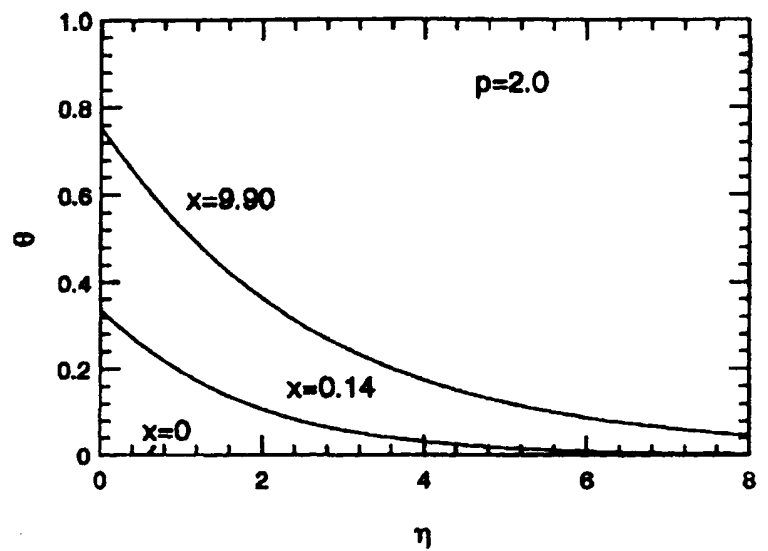

Fig. 4. Temperature profiles for $p=2.0$ axial distance $x$. This fact is in agreement with the results found by Pop and Merkin [10] for the corresponding problem of a vertical flat plate embedded in a porous medium.

The variation of the non-dimensional temperature at the outer surface of the cylinder $\theta(\bar{x}, 0)$, plotted against $\bar{x}$, is depicted in Fig. 5 for $p=0.0$ (basic case), 1.0 and 2.0, where the basic case is represented by a straight line as resulted from Eq. (17). values of $\theta(\bar{x}, 0)$ are also given in Table 1 for future reference. It is seen from this figure and Table 1 that $\theta(\bar{x}, 0)$ decreases as $p$ increases. For $p \neq 0$ this temperature is lowest at the leading edge and increases

Table 1. Representative solutions for $\theta(\bar{x}, 0)$

\begin{tabular}{llll}
\hline $\bar{x}$ & $p=0.0$ & $p=1.0$ & $p=2.0$ \\
\hline 0.0000 & 1.0000 & 0.0000 & 0.0000 \\
0.0039 & 1.0000 & 0.1829 & 0.1199 \\
0.0366 & 1.0000 & 0.3396 & 0.2326 \\
0.1434 & 1.0000 & 0.4712 & 0.3368 \\
0.3951 & 1.0000 & 0.5788 & 0.4314 \\
0.8995 & 1.0000 & 0.6654 & 0.5160 \\
1.8156 & 1.0000 & 0.7336 & 0.5904 \\
3.3754 & 1.0000 & 0.7871 & 0.6546 \\
5.9119 & 1.0000 & 0.8285 & 0.7094 \\
9.8984 & 1.0000 & 0.8608 & 0.7554
\end{tabular}

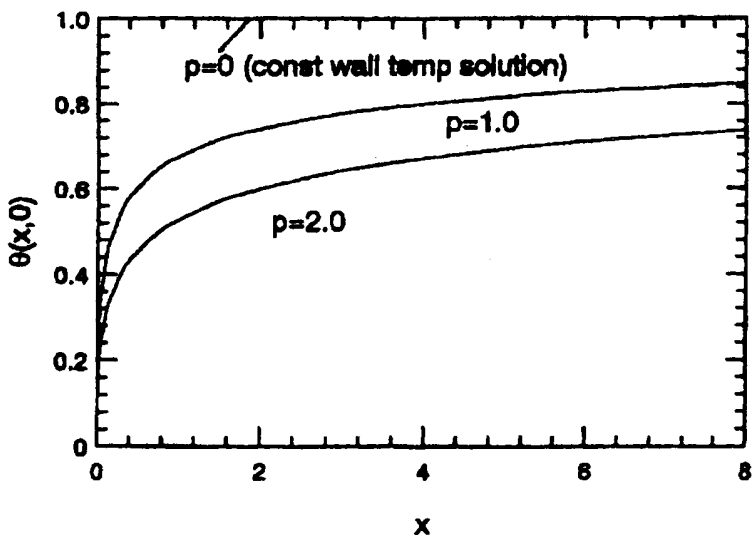

Fig. 5. Variation of $\theta(\bar{x}, 0)$ with $p$

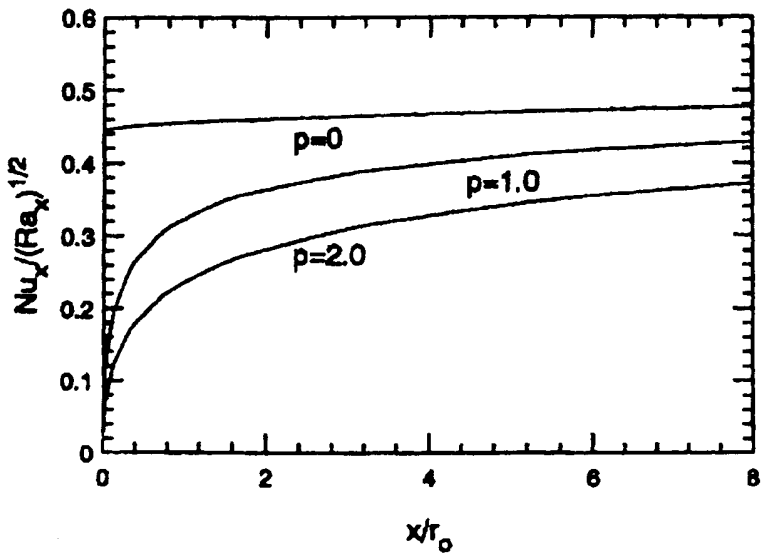

Fig. 6. Variation of $\mathrm{Nu}_{x} / \mathrm{Ra}_{x}^{1 / 2}$ with $p$ 
with $\bar{x}$ until it approaches the constant value of the basic case at very large $\bar{x}$. Finally, we show in Fig. 6 the effect of the parameter $p$ on the local Nusselt number as given by Eq. (31). Again, the basic solution $(p=0.0)$ is included into this figure. It is worth mentioning that for $p=0.0$ and $\bar{x}=0$, we found $-f^{\prime \prime}(0,0)=.44375$, while Minkowycz and Cheng [15] reported the value of 0.4440 , which shows that the present result is in excellent agreement with that from [15]. Further, it is to be noticed from Fig. 6 that the heat flux rate at the outer surface of the cylinder increases to the basic case $(p=0.0)$. This conclusion is very important for practical applications.

References

1. Nield DA; Bejan A (1992) Convection in porous media. New York, Springer

2. Ingham DB; Pop I eds. (1997) Transport phenomena in porous media. Oxford. Elsevier

3. Kimura S; Pop I (1991) Non-Darcian effects on conjugate natural convection between horizontal concentric cylinders filled with a porous medium. Fluid Dynamics Res. 7: 241-254

4. Kimura S; Pop I (1992) Conjugate natural convection between horizontal concentric cylinders filled with a porous medium. Warrne -und Stoffubertr. 85-91

5. Kimura S; Pop I (1992) Conjugate free convection from a circular cylinder in a porous medium. Int. J. Heat Mass Transfer 36: 3105-3113

6. Kimura S; Pop I (1994) Conjugate convection from a sphere in a porous medium. Int. J. Heat Mass transfer 37: 2187-2192

7. Vynnycky M; Kimura S (1994) Conjugate free convection due to a vertical plate in a porous medium. Int. J. Heat Mass Transfer 37: 229-236

8. Pop I; Lesnic, D; Ingham DB (1995) Conjugate mixed convection on a vertical surface in a porous medium. Int. J. Heat Mass transfer 38: 1917-1525

9. Lesnic D; Ingham DB; Pop I (1995) Conjugate free convection from a horizontal surface in a porous medium. J. Appl. Math Mech. (ZAMM) 75: 715-722

10. Pop I; Merkin JH (1995) Conjugate free convection on a vertical surface in a saturated porous medium. Fluid Dynam. Res. 16: 71-86

11. Vynnycky M; Kimura S (1995) Transient conjugate free convection due to a vertical plate in a porous medium. Int. J. Heat Mass Transfer 38: 219-231

12. Kimura S; Kiwata T; Okajima A; Pop I (1997) Conjugate natural convection in porous media. Adv. Water Resources 20: $111-126$

13. Keller HB (1978) Numerical methods in boundary layer theory. Ann. Rev. Fluid Mech. 10: 417-433

14. Na T-Y (1995) Effect of wall conduction on natural convection over a vertical slender hollow circular cylinder. Appl. Sci. Res. 54: 39-50

15. Minkowycz WJ; Cheng P (1995) Free convection about a vertical cylinder embedded in a porous medium. Int. J. Heat Mass Transfer 19: (1976) 805-813 